\title{
Discrete interband mutual focusing in nonlinear photonic lattices
}

\author{
Christian R. Rosberg ${ }^{1,2,3}$, Brendan Hanna ${ }^{2,3}$, Dragomir N. Neshev ${ }^{1}$, \\ Andrey A. Sukhorukov ${ }^{1,3}$, Wieslaw Krolikowski ${ }^{2,3}$, \\ and Yuri S. Kivshar ${ }^{1,3}$ \\ ${ }^{1}$ Nonlinear Physics Centre, ${ }^{2}$ Laser Physics Centre, and ${ }^{3}$ Centre for Ultrahigh-bandwidth \\ Devices for Optical Systems (CUDOS), Research School of Physical Sciences and \\ Engineering, Australian National University, Canberra, ACT 0200, Australia \\ http://www.rsphysse.anu.edu.au/nonlinear
}

\begin{abstract}
We study nonlinear coupling of mutually incoherent beams associated with different Floquet-Bloch waves in a one-dimensional optically-induced photonic lattice. We demonstrate experimentally how such interactions lead to asymmetric mutual focusing and, for waves with opposite diffraction properties, to simultaneous focusing and defocusing as well as discreteness-induced beam localization and reshaping effects.
\end{abstract}

(C) 2005 Optical Society of America

OCIS codes: (190.4420) Nonlinear optics, transverse effects in; (190.5940) Self-action effects

\section{References and links}

1. Yu. S. Kivshar and G. P. Agrawal, Optical Solitons: From Fibers to Photonic Crystals (Academic Press, San Diego, 2003).

2. H. S. Eisenberg, Y. Silberberg, R. Morandotti, and J. S. Aitchison, "Diffraction management," Phys. Rev. Lett. 85, 1863-1866 (2000).

3. H. S. Eisenberg, Y. Silberberg, R. Morandotti, A. R. Boyd, and J. S. Aitchison, "Discrete spatial optical solitons in waveguide arrays," Phys. Rev. Lett. 81, 3383-3386 (1998).

4. J. W. Fleischer, T. Carmon, M. Segev, N. K. Efremidis, and D. N. Christodoulides, "Observation of discrete solitons in optically induced real time waveguide arrays," Phys. Rev. Lett. 90, 023902-4 (2003).

5. D. Neshev, E. Ostrovskaya, Y. Kivshar, and W. Krolikowski, "Spatial solitons in optically induced gratings," Opt. Lett. 28, 710-712 (2003).

6. J. W. Fleischer, M. Segev, N. K. Efremidis, and D. N. Christodoulides, "Observation of two-dimensional discrete solitons in optically induced nonlinear photonic lattices," Nature 422, 147-150 (2003).

7. D. Mandelik, R. Morandotti, J. S. Aitchison, and Y. Silberberg, "Gap solitons in waveguide arrays," Phys. Rev. Lett. 92, 093904-4 (2004).

8. D. Neshev, A. A. Sukhorukov, B. Hanna, W. Krolikowski, and Yu. S. Kivshar, "Controlled generation and steering of spatial gap solitons," Phys. Rev. Lett. 93, 083905-4 (2004).

9. O. Cohen, T. Schwartz, J. W. Fleischer, M. Segev, and D. N. Christodoulides, "Multiband vector lattice solitons," Phys. Rev. Lett. 91, 113901-4 (2003).

10. A. A. Sukhorukov and Yu. S. Kivshar, "Multigap discrete vector solitons," Phys. Rev. Lett. 91, 113902-4 (2003).

11. H. Buljan, O. Cohen, J. W. Fleischer, T. Schwartz, M. Segev, Z. H. Musslimani, N. K. Efremidis, and D. N. Christodoulides, "Random-phase solitons in nonlinear periodic lattices," Phys. Rev. Lett. 92, 223901-4 (2004).

12. K. Motzek, A. A. Sukhorukov, F. Kaiser, and Yu. S. Kivshar, "Incoherent multi-gap optical solitons in nonlinear photonic lattices," Opt. Express 13, 2916-2923 (2005).

13. O. Cohen, G. Bartal, H. Buljan, T. Carmon, J. W. Fleischer, M. Segev, and D. N. Christodoulides, "Observation of random-phase lattice solitons," Nature 433, 500-503 (2005).

14. D. Mandelik, H. S. Eisenberg, Y. Silberberg, R. Morandotti, and J. S. Aitchison, "Observation of mutually trapped multiband optical breathers in waveguide arrays," Phys. Rev. Lett. 90, 253902-4 (2003).

15. Y. Lahini, D. Mandelik, Y. Silberberg, and R. Morandotti, "Polarization dependent properties of waveguide arrays: band-structure anomaly and high-band localizations," Opt. Express 13, 1762-1773 (2005).

16. S. Trillo, S. Wabnitz, E. M. Wright, and G. I. Stegeman, "Optical solitary waves induced by cross-phase modulation," Opt. Lett. 13, 871-873 (1988). 
17. V. V. Afanasyev, Yu. S. Kivshar, V. V. Konotop, and V. N. Serkin, "Dynamics of coupled dark and bright optical solitons," Opt. Lett. 14, 805-807 (1989).

18. Yu. S. Kivshar, D. Anderson, A. Hook, M. Lisak, A. A. Afanasjev, and V. N. Serkin, "Symbiotic optical solitons and modulational instability," Phys. Scr. 44, 195-202 (1991).

19. N. K. Efremidis, S. Sears, D. N. Christodoulides, J. W. Fleischer, and M. Segev, "Discrete solitons in photorefractive optically induced photonic lattices," Phys. Rev. E 66, $046602-5$ (2002).

\section{Introduction}

Light propagation in nonlinear media is associated with many important phenomena including optical rectification, harmonic generation, self-focusing, and soliton formation. Coupling of two or more optical waves mediated by a nonlinear material is responsible for four-wave mixing, parametric generation and amplification, as well as cross phase modulation and cross coupling. Mutual beam focusing involves both self-focusing and cross phase modulation of co-propagating beams and is an important effect with potential for all-optical light control and switching applications. Mutual beam focusing is also a fundamental phenomenon responsible for the formation of vector optical solitons in homogeneous dielectric media [1].

Periodicity of the medium adds to the diversity of effects associated with nonlinear light propagation. In a material with a periodic modulation of the refractive index, the linear transmission spectrum becomes subject to dramatic modifications such as for instance the appearence of bandgaps, and the light propagation displays many new features that are governed by the properties of the extended eigenmodes of the periodic structure, the Floquet-Bloch waves. For example, beams associated with different Floquet-Bloch modes possess different diffraction properties, and this leads to normal, zero, and even anomalous diffraction in the same material [2]. Furthermore, the bandgap spectrum of a periodic medium strongly affects nonlinear propagation and localization of light in the form of spatial solitons [1]. Different types of such self-trapped optical wavepackets have been demonstrated experimentally in nonlinear periodic structures, including discrete (or lattice) solitons in the semi-infinite total internal reflection gap [3, 4, 5], and spatial gap solitons in the Bragg reflection gaps [4, 6, 7, 8]. Vector solitons consisting of mutually coupled components localized in different gaps have been predicted theoretically $[9,10,11,12]$. Most recently, it was demonstrated experimentally that partially coherent beams can excite modes in several gaps that experience mutual trapping in media with slow photorefractive nonlinearity [13]. Mutual focusing of orthogonally polarized waves excited in different bands, as well as formation of single-polarization multi-band breathers, has been observed in AlGaAs waveguide array structures [14, 15].

In this work, we study experimentally nonlinear interactions of mutually incoherent beams associated with different Floquet-Bloch modes of an optically-induced photonic lattice with self-focusing nonlinearity. The setup was designed to allow for simultaneous excitation of waves associated with the edges of the first and second transmission bands, and for independent control over the beam widths and amplitudes. Such flexibility enabled us to reveal novel effects for nonlinear beam propagation regimes that were not accessed in other experimental studies of interband mutual focusing $[13,14,15]$. In particular, we demonstrate an inherent modal asymmetry in the effect of discrete mutual focusing of beams generated at the upper edges of the first two transmission bands. We observe transitional mutual focusing and defocusing of waves exhibiting diffraction of different magnitude and sign, and study in detail discrete beam break-up induced by highly localized fields. The results allow for generalization of symbiotic type interactions in periodic media, as predicted for pulses with different dispersion in optical fibres $[16,17,18]$

We consider a one-dimensional lattice induced optically in a photorefractive crystal with strong electro-optic anisotropy, as first suggested theoretically by Efremidis et al. [19]. Propagation of several mutually incoherent beams in such a lattice is described by a system of coupled

\#7698 - \$15.00 USD

(C) 2005 OSA
Received 6 June 2005; revised 24 June 2005; accepted 27 June 2005 11 July 2005 / Vol. 13, No. 14 / OPTICS EXPRESS 5370 
nonlinear Schrödinger equations for the normalized beam envelopes $E_{n}(x, z)$,

$$
i \frac{\partial E_{n}}{\partial z}+D \frac{\partial^{2} E_{n}}{\partial x^{2}}+\mathscr{F}(x, I) E_{n}=0
$$

where $I=\sum_{n}\left|E_{n}\right|^{2}$ is the total intensity, $x$ and $z$ are the transverse and propagation coordinates normalized to the characteristic values $x_{0}$ and $z_{0}$, respectively, $D=z_{0} \lambda_{0} /\left(4 \pi n_{0} x_{0}^{2}\right)$ is the beam diffraction coefficient, $n_{0}$ is the average refractive index of the medium, and $\lambda_{0}$ is the wavelength in vacuum. The optically-induced refractive index change is $\mathscr{F}\left(x,|E|^{2}\right)=$ $-\gamma\left(I_{b}+I_{p}(x)+|E|^{2}\right)^{-1}$, where $I_{b}$ is the constant dark irradiance, $I_{p}(x)=I_{g} \cos ^{2}(\pi x / d)$ is the interference pattern which induces a lattice with period $d$, and $\gamma$ is a nonlinear coefficient proportional to the applied DC field $[4,5]$. To match our experimental conditions, we use the following parameters: $\lambda_{0}=0.532 \mu \mathrm{m}, n_{0}=2.35, x_{0}=1 \mu \mathrm{m}, z_{0}=1 \mathrm{~mm}, d=19.2, I_{b}=1$ and $I_{g}=1$. Then, the refractive index contrast in the lattice is $\Delta n=\gamma \lambda /\left(4 \pi z_{0}\right)$, which evaluates to $1 \cdot 10^{-4}$ for $\gamma=2.36$. The crystal length is $L=15 \mathrm{~mm}$.

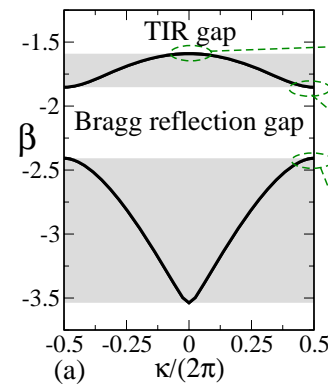

(a)

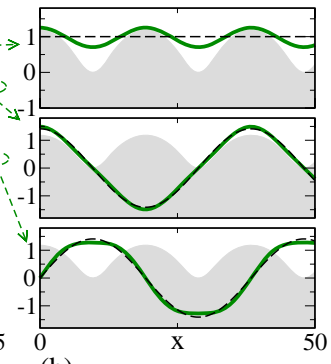

(b)

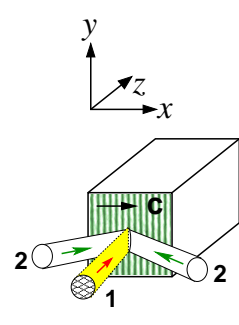

(c)

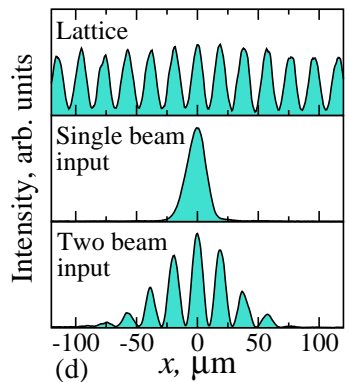

(d)

Fig. 1. (a) Dispersion of the Bloch waves in an optically-induced lattice; the bands are shaded. (b) Bloch-wave profiles at the gap edges (solid) and their leading-order Fourier components (dashed), superimposed on the normalized refractive index profile (shaded). (c) Schematic of (1) the Bloch-wave excitation with a single beam and (2) a pair of beams inclined at the Bragg angle. (d) Experimental intensity profiles of the optical lattice and the input beams.

\section{Experimental setup}

We create an optical lattice with a period of $19.2 \mu \mathrm{m}$ inside a $15 \times 5 \times 5 \mathrm{~mm}$ SBN: 60 crystal by interfering two ordinarily-polarized broad laser beams from a cw frequency-doubled $\mathrm{Nd}: \mathrm{YVO}_{4}$ laser at $532 \mathrm{~nm}$. Applying an external electric field of $1.8-2.0 \mathrm{kV}$ then produces a periodic refractive index modulation whose saturation is controlled by homogeneously illuminating the crystal with white light. The induced lattice gives rise to a linear transmission spectrum with bandgaps as illustrated in Fig. 1(a). Extraordinarily-polarized probe beams from the same laser source are focused onto the front face of the crystal and launched into the lattice in a way as to selectively excite Bloch waves associated with the edges of the bandgaps. The eigenmode profiles at the gap edges are shown in Fig. 1(b) along with the corresponding refractive index modulation (shaded).

In order to excite the specific Bloch waves, we use a multiple-beam technique, schematically shown in Fig. 1(c). To obtain pure excitation of a given Bloch wave, the input beam must approximate the eigenmode profile by matching its leading-order Fourier component [Fig. 1(bdashed line)]. Hence, a single Gaussian beam [Fig. 1(d-middle)] is used to excite the fundamental wave at the top of the first band (i.e. at the edge of the total internal reflection gap) [5], and two overlapping coherent beams, both inclined at the Bragg angle, are used to excite Bloch waves with a staggered phase structure at the two edges of the Bragg reflection gap [8]. 
Aligning the maxima of the lattice and the two-beam interference profile [Fig. 1(d-bottom)], we obtain pure excitation of the Bloch mode at the bottom of the first band. Shifting the probe beam position by half a period leads to a pure excitation of the mode at the top of the second band. The beams 1 and 2 in Fig. 1(c) are made mutually incoherent by reflecting beam 1 from a piezo-transducer controlled mirror vibrating at high frequency (a few hundred $\mathrm{Hz}$ ). All excited Bloch waves propagate straight down the lattice with zero transverse velocity, in case of the staggered modes thanks to an exact power balance between the two inclined input beams. At the crystal output, the near-field intensity distribution of the propagated beams is imaged onto a CCD camera. Since the nonlinear response time of the crystal is in the order of several seconds, each probe beam output can be recorded separately by blocking the other probe and optical lattice beams immediately $(<1 \mathrm{~s})$ before taking a snapshot.

\section{Discrete interband mutual focusing}

We first study discrete interband coupling of two beams associated with the top of the first and the second band. Both exhibit normal diffraction and can therefore be localized at higher powers by the self-focusing nonlinearity. This leads to the effect of mutual focusing since each beam is capable of inducing a local defect (waveguide) that traps the other component. Mutual focusing can occur for a wide range of different relative and absolute beam powers. Here we investigate the extreme cases in which the beam powers are strongly imbalanced, and where the coupling effects are therefore most easily observed. We initially set the power of both beams at a low $(\mathrm{nW})$ level so that they propagate in a linear regime. Then the power of one of the components is increased and the nonlinear effect on both beams is studied. Unless otherwise stated, the two probe beams have an input full width at half maximum (FWHM) of $17 \pm 3 \mu \mathrm{m}$ and $70 \pm 3 \mu \mathrm{m}$, respectively. All beam powers are measured as the total output from the crystal back face.
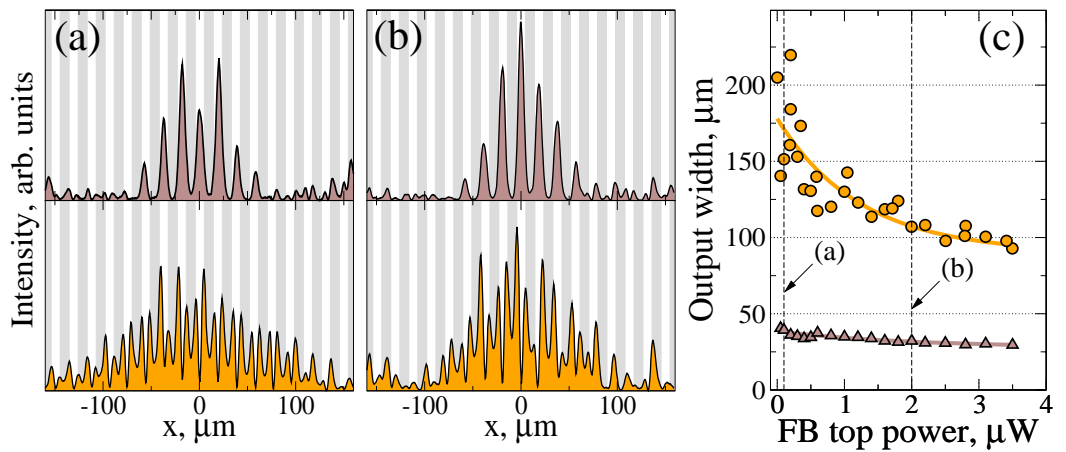

Fig. 2. Experimental results for interband mutual focusing of the beams generated at the top of the $2^{\text {nd }}$ band (orange) and top of the $1^{\text {st }}$ band (brown). (a) Both components at low power. (b) $1^{\text {st }}$-band beam at high power and $2^{\text {nd }}$-band beam at low power $(70 \mathrm{nW})$. (c) Beam width vs. power of the $1^{\text {st }}$-band mode. Solid curves are exponential fits to the data points.

Figure 2 shows the result of increasing the power of the fundamental first band beam (brown). Transition from discrete diffraction of this beam in the linear regime [Fig. 2(a)] to discrete focusing at higher power [Fig. 2(b)] is accompanied by a decrease in the FWHM of the broader co-propagating second-band beam by almost a factor of two. The second-band beam width is estimated by a Gaussian envelope fitting, whereas the width of the first-band beam is described by its discrete second-order central moment $w^{2}=\sum 2^{|n|} d^{2} P(n) / \sum P(n)$, where $2 n+1$ is the number of spanned lattice sites, $d$ is the lattice period, and $P(n)$ is the intensity of the $n^{\text {th }}$ site. 
We calculate $w^{2}$ for the three central lattice sites $(n=1)$ and characterize the beam width by $2 w$. To investigate the effect in further detail, we measure the degree of focusing of both components as a function of the first-band beam power. The result is shown in Fig. 2(c), and we notice that the width of the passive second-band beam asymptotically shrinks from $\sim 180 \mu \mathrm{m}$ to $\sim 90 \mu \mathrm{m}$, whereas the effect is rather small for the first-band beam itself.
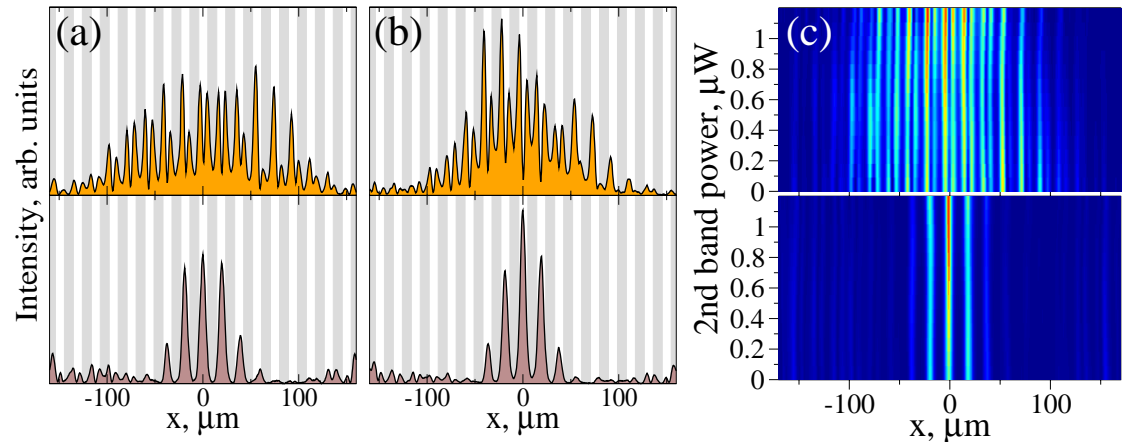

Fig. 3. Experimental results for interband mutual focusing of the beams generated at the top of the $1^{\text {st }}$ band (brown) and top of the $2^{\text {nd }}$ band (orange). (a) Both components at low power. (b) $2^{\text {nd }}$-band beam at high power $(900 \mathrm{nW})$ and $1^{\text {st }}$-band beam at low power $(100 \mathrm{nW})$. (c) Two-dimensional visualization of output profiles for increasing $2^{\text {nd }}$-band power.

Simultaneous beam focusing is also observed if, conversely, the power of the first-band component is maintained at a low level and that of the second band beam is increased [Fig. 3]. Since the defect induced by the high-power second-band beam is rather broad and weak, we observe less pronounced focusing of the first-band beam in this case. In fact, it was necessary to slightly increase the input beam width to $20 \mu \mathrm{m}$ in order to reduce diffraction and thereby facilitate focusing, and that is why no clear discrete diffraction is observed for linear propagation of the first-band beam in Fig. 3(a). Figure 3(c) maps the beam profiles as a function of the second-band beam power in a two-dimensional plot.

Together the above results demonstrate discrete interband mutual focusing of mutually incoherent beams, an effect responsible for the formation of multi-gap vector solitons. The observed asymmetry in the strength of the coupling between the bands reflects the fact that the physics of the discrete total internal reflection and Bragg gap solitons associated with the involved Bloch waves is quite different. Indeed, a discrete soliton in the total internal reflection gap creates a narrow and strongly localized defect, whereas both the minimum width and the maximum intensity of a Bragg gap soliton are limited due to the finite spectral width of the Bragg reflection gap. This means that there is also a limit for the trapping strength of the self-induced secondband defect. The above-mentioned generic features may well account for the saturation of the second band mode focusing [Fig. 2(c)], and the observed interband coupling asymmetry, i.e. the fact that the two beams are not equally capable of trapping each other.

\section{Interaction of beams with normal and anomalous diffraction}

Next we study intraband coupling effects for the two modes at the top and the bottom of the first band. These waves exhibit normal and anomalous diffraction, respectively, and therefore the first self-focuses while the second experiences defocusing in the self-focusing nonlinearity. However, the picture is more complicated when the two waves interact via the nonlinear medium. We show that in this case mutual coupling can lead to simultaneous focusing and defocusing and to interesting discrete localization effects. Indeed, when the power of the beam associated with the bottom of the band is increased [Fig. 4] it does spread out stronger due to 
self-defocusing (blue). However, it still induces a lattice defect that causes the mode at the top of the band (brown) to focus. This is to our knowledge a first demonstration of simultaneous mutual focusing and defocusing of beams belonging to different parts of the bandgap spectrum. Figure 4(c) shows that the beam widths increase and decrease almost linearly as a function of the power of the beam at the top of the band. We would like to point out that whereas the interband mutual focusing can lead to the formation of stable multi-gap vector solitons, the intra-band coupling effects responsible for the observed simultaneous focusing and defocusing [Fig. 4] are inherently transient in nature since at longer propagation distances, the two beams will eventually decouple from each other. However, soliton formation is not in general required for obtaining e.g. all-optical switching, and even spatially transient effects may be sufficient for achieving effective beam manipulation functionalities.
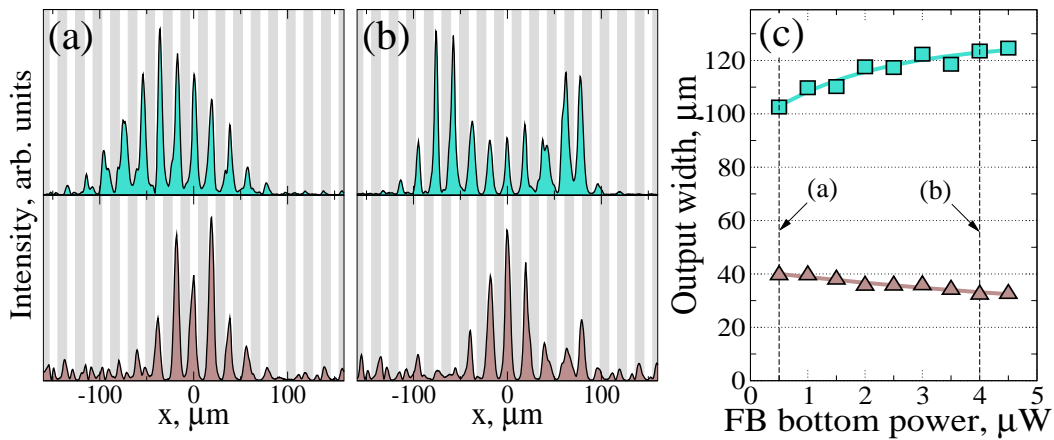

Fig. 4. Experimental results for coupling of beams at the top (brown) and bottom (blue) of the $1^{\text {st }}$ band. (a) Both components at low power. (b) Bottom of $1^{\text {st }}$ band at high power and top of $1^{s t}$ band at low power $(100 \mathrm{nW})$. (c) Beam width vs. power of the $1^{\text {st }}$ band bottom mode.
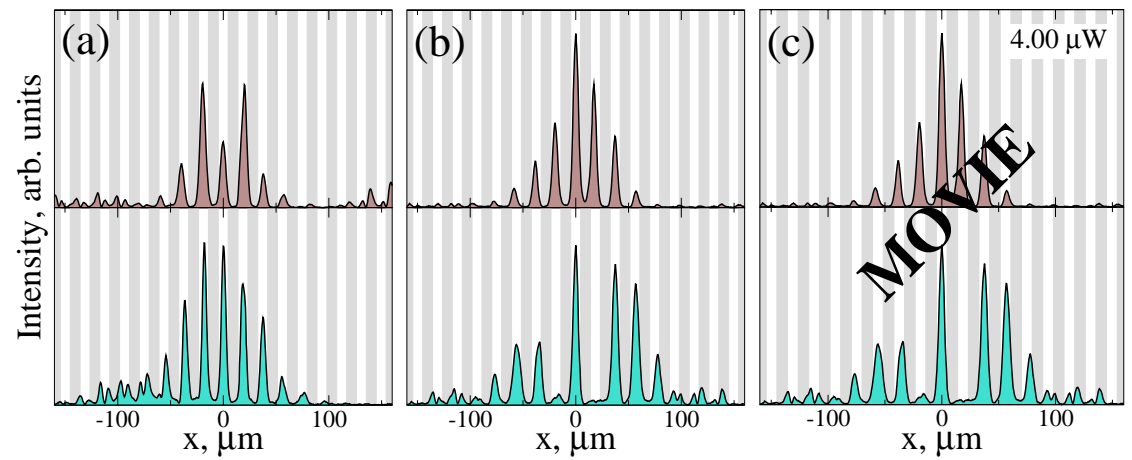

Fig. 5. Experimental results for coupling of beams at the bottom (blue) and top (brown) of the $1^{\text {st }}$ band. (a) Both components at low power $(50 \mathrm{nW})$. (b) Top of $1^{\text {st }}$ band at high power $(4.0 \mu \mathrm{W})$ and bottom of $1^{s t}$ band at low power. (c) Movie $(0.4 \mathrm{Mb})$ shows the fundamental beam self-focusing and the simultaneous appearance of a discrete localized peak and two decoupled outer lobes for the beam at the bottom of the band. The power of the fundamental beam is shown in the upper right corner.

In the opposite case [see Fig. 5], the beam at the top of the band self-focuses at high powers, but induces a repelling defect for the defocusing beam at the bottom of the band. We observe that as the power of the fundamental beam is increased [movie in Fig. 5(c)] the power carried by the two lattice sites (index maxima) next to the central one drops significantly at the crystal 
output. Eventually, at high power, the output profile is composed of a single centrally localized peak and two discretely repelled outer lobes. Clearly, this complex beam reshaping cannot be described by a simple envelope approximation and calls for further investigations.

\section{Numerical simulations}

To support our experimental results and to more closely examine the features associated with discrete localization and beam shaping effects for waves with opposite diffraction properties, a series of extended numerical simulations were performed. They confirmed all important aspects of our experimental observations, including the asymmetric interband coupling, the transitional mutual focusing and defocusing, and the discreteness-induced reshaping of the anomalously diffracting first-band beam. Fig. 6 shows two examples of simulations representing the experimental cases of mutual interband focusing in Fig. 2 and first band focusing-defocusing interactions in Fig. 5. The simulated propagation distance corresponds to the length of our crystal.

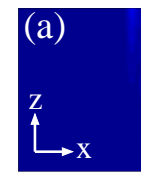

(e)

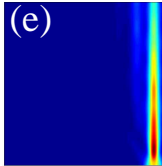

Top of 1 st band

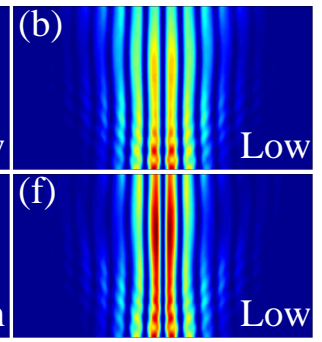

Top of 2 nd band
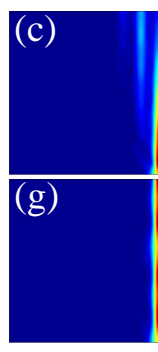

Top of 1 st band Bottom of 1 st band

Fig. 6. Beam propagation method simulations of interband focusing (left) and interaction of focusing and defocusing $1^{\text {st }}$-band beams (right). The plot windows are $320 \mu \mathrm{m}$ and $15 \mathrm{~mm}$ in the transverse (x) and propagation (z) directions, respectively. In (a,b) and (c,d) two beams co-propagate at low power and experience diffraction. In $(e, f)$ the power of the beam at the top of the $1^{\text {st }}$ band is increased, causing focusing of this beam and the low power $2^{\text {nd }}$-band beam. In $(\mathrm{g}, \mathrm{h})$ interaction between a high power fundamental beam and a low power beam at the bottom of the $1^{\text {st }}$ band results in the formation of a complex beating pattern and the appearance of a localized discrete central peak and two diffracting outer lobes.

The first case [Fig. 6, left] presents a pair consisting of a fundamental and a second band beam which both experience diffraction broadening at low power (top panels) and then focus as the power of the first band component is increased (bottom panels). Figure 6 (right) shows a pair of beams with opposite diffraction from the top and bottom of the first band. At low power, both components propagate linearly and diffract. At high power, the fundamental mode (top of the band) self-focuses and induces a local lattice defect, which results in a complex propagation pattern for the anomalously diffracting wave at the bottom of the band. First, power seems to oscillate back and forth between the central lattice site and its two adjacent neighbours, indicating a beating between modes with different propagation constants. Then, a central peak and two clearly decoupled outer lobes eventually appear, as observed experimentally. Simulations for extended propagation distances (not shown) revealed that after about 50\% longer propagation the oscillatory behavior ceases, and the decoupled power propagates away from the defect in two distinct lobes at the zero diffraction angles. Interestingly, a considerable part of the power stays trapped in the induced defect and propagates straight down the lattice as a stable waveguide mode. In all simulations the FWHMs of the fundamental and the Bragg input beams were $15 \mu \mathrm{m}$ and $70 \mu \mathrm{m}$, respectively, and the (high) peak power of the fundamental 
first-band beam (normalized to a constant dark irradiance $I_{b}$ ) was 0.25 and 3.24 in Figs. 6 (e,f) and $(\mathrm{g}, \mathrm{h})$, respectively.

\section{Conclusions}

In conclusion, we have studied interaction of mutually incoherent beams associated with Floquet-Bloch waves from different spectral bands. We have demonstrated experimentally how defects induced by nonlinear propagation can trap and guide beams from several bands, leading to the effect of discrete interband mutual focusing. This is a key physical mechanism for generation of multi-gap solitons with components originating from different spectral bands. We have observed a fundamental asymmetry in the interband mutual focusing effect due to the generically different physics of the involved Bloch waves. We also demonstrated an interplay between waves with diffraction coefficients of opposite sign that leads to generation of anti-waveguiding defects, complex beam reshaping and simultaneous focusing and defocusing within the first transmission band. 